\title{
Couverture de la population dans le Système canadien de surveillance des maladies chroniques : enquête sur le contenu des registres d'assurance-maladie au Canada
}

Naomi C. Hamm, M. Sc. (1); Cynthia Robitaille, M. Sc. (2); Joellyn Ellison, M.S.P. (2); Siobhan O’Donnell, M. Sc. (2); Louise McRae, B. Sc. (2); Kimberley Hutchings, M. Sc. (2); Louis Rochette, M. Sc. (3); Karen A.M. Phillips, D.M.V. (4); Mahmoud Azimaee, B. Sc. (5); MaryRose Stang, Ph. D. (6); Rolf Puchtinger, M.A. (6); Megan McCallum, MSP (7); Aijun Yang, M. Sc. (8); Josh Squires, B. Sc. (9); Yue Liu, M. Ing. (10); Lawrence W. Svenson, Ph. D. (11,12,13); Faisal Shibley, B. Sc. (14); Aakash Amatya, M. Sc. (14); Bin Zhang, Ph. D. (15); James Ayles, B. Sc. (15); Lisa M. Lix, Ph. D. (1)

Cet article a fait l'objet d'une évaluation par les pairs.

\section{Résumé}

Introduction. Les registres d'assurance-maladie, qui recueillent des renseignements sur la couverture et certaines caractéristiques des individus à l'échelle de larges populations, sont précieux pour la surveillance de la santé de la population et de la recherche fondée sur l'utilisation de données administratives. Le fait que les données des registres des provinces et des territoires du Canada ne soient pas normalisées est susceptible de nuire à la comparabilité des mesures de surveillance. Nous avons évalué le contenu des registres d'assurance-maladie au Canada afin de décrire les populations couvertes et de relever les similitudes et les différences entre les registres.

Méthodologie. L'équipe de l'étude et des représentants de l'Agence de la santé publique du Canada ont élaboré une enquête sur les caractéristiques individuelles et les données sur la population recueillies dans les registres d'assurance-maladie. Les réponses des informateurs clés des provinces et territoires qui ont répondu à l'enquête ont été analysées de façon descriptive.

Résultats. Toutes les provinces ont répondu à l'enquête et les Territoires du Nord-Ouest ont fourni des réponses partielles. Certaines caractéristiques individuelles figurant dans les registres d'assurance-maladie (l'adresse principale, la date de naissance et le sexe) sont saisies dans toutes les provinces et tous les territoires. Les données disponibles sur les liens de parenté, l'origine ethnique et le statut socioéconomique sont variables selon les provinces et territoires, tout comme les dates de début et de fin de couverture et la fréquence de mise à jour du registre. L'information sur des populations particulières, comme les membres des Premières Nations, est saisie dans certaines provinces et certains territoires seulement.

Suite à la page suivante

Rattachement des auteurs :

1. Département des sciences de la santé communautaire, Université du Manitoba, Winnipeg (Manitoba), Canada

2. Agence de la santé publique du Canada, Ottawa (Ontario), Canada

3. Bureau d'information et d'études en santé des populations, Institut national de santé publique du Québec, Montréal

(Québec), Canada

4. Bureau de l'administrateur en chef de la santé publique, ministère de la Santé et du Bien-être de l'île-du-Prince-Édouard,

Charlottetown (Île-du-Prince-Édouard), Canada

5. Data Quality and Information Management, ICES, Toronto (Ontario), Canada

6. Direction générale de la santé de la population, ministère de la santé de la Saskatchewan, Regina (Saskatchewan), Canada

7. Gouvernement des Territoires du Nord-Ouest, Yellowknife (Territoires du Nord-Ouest), Canada

8. Ministère de la Santé de la Colombie-Britannique, Victoria (Colombie-Britannique), Canada

9. Services de données et d'information, Centre d'information sur la santé de Terre-Neuve-et-Labrador, St. John's (Terre-Neuve-et-Labrador), Canada

10. Ministère de la Santé et du Mieux-être de la Nouvelle-Écosse, Halifax (Nouvelle-Écosse), Canada

11. Analytics and Performance Reporting, Alberta Health, Edmonton (Alberta), Canada

12. Division de la médecine préventive, École de santé publique, Université de l'Alberta, Edmonton (Alberta), Canada

13. Département des sciences de la santé communautaire, Université de Calgary, Calgary (Alberta), Canada

14. Ministère de la Santé, des Aînés et de la Vie active, gouvernement du Manitoba, Winnipeg (Manitoba), Canada

15. Ministère de la Santé du Nouveau-Brunswick, Fredericton (Nouveau-Brunswick), Canada

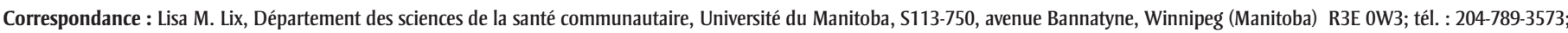
téléc. : 204-789-3905; courriel : lisa.lix@umanitoba.ca
Points saillants

- Tous les registres canadiens de déclaration de l'assurance-maladie contiennent des données remontant au moins à 1996. La première année pour laquelle des données sont disponibles est 1968, en Saskatchewan.

- Certaines données fournies par le registre, comme la modification de la couverture, l'adresse principale, la date de naissance et le sexe des individus, sont uniformes dans toutes les administrations déclarantes. D'autres caractéristiques, telles que l'information sur les relations familiales, le statut socioéconomique et d'autres données sur les individus, sont variables.

- Les différences entre les registres d'assurance-maladie canadiens permettent d'effectuer des comparaisons entre les populations couvertes par les régimes d'assurance-maladie provinciaux et territoriaux. La création d'un cadre de données harmonisé serait, quant à elle, utile aux stratégies de surveillance nationales et aux études intergouvernementales. 
Conclusion. Les registres d'assurancemaladie constituent une riche source d'information sur les populations assurées des provinces et des territoires. Cependant, l'hétérogénéité des données peut avoir un impact sur les personnes incluses et exclues dans les estimations de surveillance de la population produites à partir de données administratives sur la santé. L'élaboration d'un cadre de données harmonisé favoriserait l'obtention de résultats comparables et à jour en matière de recherche et de surveillance de la santé de la population dans le cadre d'études intergouvernementales.

Mots-clés : registres d'assurance-maladie, données administratives sur la santé, identificateurs de population.

\section{Introduction}

Les données administratives sur la santé sont recueillies à l'origine à des fins de gestion et de surveillance du système de soins de santé. Cependant, elles sont aussi souvent utilisées pour mesurer et décrire la santé de la population à l'échelle du Canada $^{1-7}$ car elles sont recueillies régulièrement, qu'il est peu coûteux de les utiliser et qu'elles couvrent presque toute la population. Les données administratives sur la santé sont les demandes de paiement des médecins, les registres de sortie des hôpitaux, les ordonnances de médicaments et les registres d'assurancemaladie $^{8}$. Les registres d'assurance-maladie sont précieux à la fois pour la surveillance à l'échelle des populations et pour les études de recherche au Canada, car ils contiennent des données sur les populations provinciales et territoriales admissibles aux services de santé financés par l'État ${ }^{9}$. Il est essentiel de comprendre quelles populations sont inscrites dans les registres d'assurance-maladie et quelles données sont recueillies sur ces populations afin d'être capable de comparer les mesures de santé entre provinces et territoires et au fil du temps et de déterminer si ces comparaisons sont généralisables.

Au Canada, la couverture des soins de santé relève principalement des provinces et territoires ${ }^{10}$. Il n'existe aucune norme sur la façon dont les données des registres d'assurance-maladie sont recueillies et codées, ni sur la façon dont les différentes populations, dont celles couvertes par l'assurance-maladie fédérale, sont identifiées dans les fichiers des registres. Les différences entre registres d'assurance-maladie au Canada sont susceptibles d'entraîner des difficultés lors des comparaisons dans les études de surveillance et de recherche.

Le Système canadien de surveillance des maladies chroniques (SCSMC) est un réseau appuyé par l'Agence de la santé publique du Canada (ASPC) qui vise à favoriser l'utilisation des données administratives canadiennes sur la santé pour surveiller des maladies chroniques et qui vise également à contribuer à la planification des soins de santé ainsi que les politiques et les programmes de santés. Par l'entremise du SCSMC, les provinces et territoires transmettent à l'ASPC des données agrégées tirées des bases de données administratives sur la santé, qui sont ensuite utilisées pour produire des estimations nationales et des tendances au fil du temps pour plus de vingt maladies et affections chroniques. L'hétérogénéité des données contenues dans les registres d'assurance-maladie risque d'avoir un impact sur les personnes incluses dans les estimations du SCSMC et celles qui en sont exclues. Elle a également des répercussions sur les provinces et territoires dans leurs recherches en santé de souspopulations particulières (par exemple les membres des Premières Nations) ainsi que dans les activités de surveillance à cet égard.

Peu de recherches ont été menées sur les données des registres d'assurance-maladie des provinces et territoires canadiens. Les rares études disponibles portent principalement sur les registres de l'assurance-maladie d'une province ou d'un territoire spécifique. Deux études portent sur le registre de l'assurance-maladie du Manitoba ${ }^{9,11}$ et une autre décrit brièvement le registre du Québec dans le cadre du Système intégré de surveillance des maladies chroniques de cette province ${ }^{5}$. Les études qui portent sur plusieurs provinces et territoires se limitent à des examens, aucune comparaison directe n’ayant été faite entre provinces et territoires ${ }^{12,13}$. Des détails à propos de l'information disponible sur les populations et le fait de savoir quelles personnes sont incluses dans les registres d'assurance-maladie fondés sur la population et quelles sont celles qui en sont exclues pourraient (1) améliorer notre compréhension des estimations de la surveillance produites à partir des données du SCSMC et (2) préciser les utilisations potentielles des données des registres pour la description de sous-populations particulières.

Cette étude a donc pour objet d'évaluer les données des registres d'assurancemaladie des provinces et territoires canadiens. Elle vise à décrire les populations couvertes et à relever les similitudes et les différences entre les registres.

\section{Méthodologie}

Les données ont été recueillies à l'aide d'une enquête que l'équipe du projet a élaborée en collaboration avec des experts en contenu et des conseillers de l'ASPC. De plus, l'équipe du projet a reçu les commentaires du personnel de recherche du Centre d'élaboration de la politique des soins de santé du Manitoba, qui a déjà travaillé avec les données de population d'un registre d'assurance-maladie. L'enquête comprenait cinq sections : (1) les renseignements généraux (c.-à-d. la province ou le territoire), (2) la couverture temporelle des données, (3) les dates de début et de fin de la couverture des résidents de la province ou du territoire, (4) les caractéristiques individuelles consignées dans le registre et (5) l'information disponible sur la population. L'ASPC a approuvé l'ébauche finale de l'enquête avant qu'elle ne soit distribuée. Il est possible d'obtenir un exemplaire de l'enquête sur demande.

L'équipe du projet, avec l'aide de l'ASPC, a dressé une liste d'informateurs clés pour chaque province et territoire. Ces informateurs ont été ciblés principalement parmi les membres du Comité scientifique du SCSMC et du Groupe de travail sur la qualité des données. Nous avons communiqué avec des informateurs qui occupaient des postes exigeant de travailler avec des données administratives, car ces derniers étaient les plus susceptibles de connaître les données des registres de leur province ou territoire. Dans les cas où deux personnes-ressources possédant une expertise semblable étaient disponibles, nous avons communiqué avec les deux.

L'équipe du projet a envoyé le questionnaire d'enquête par courriel aux principaux répondants en avril 2019. Ces derniers disposaient d'une semaine pour y répondre, avec extension de délai au besoin. Les informateurs clés pouvaient, en cas de besoin, consulter d'autres experts de leur province ou territoire afin de répondre à l'enquête et, s'ils ne pouvaient pas y répondre, ils étaient invités à recommander 
un autre informateur. L'équipe du projet a effectué des suivis par téléphone et par courriel afin de préciser les réponses lorsque c'était nécessaire.

Cette étude a été menée hors de l'Université du Manitoba. Il n’a pas été nécessaire d'obtenir l'approbation éthique de la recherche, car les données recueillies portaient sur le contenu du registre de l'assurance-maladie et non sur des participants humains ${ }^{14}$.

\section{Résultats}

Treize informateurs clés des dix provinces ont répondu à l'enquête (deux pour la Saskatchewan, le Manitoba et le NouveauBrunswick) et un informateur clé des Territoires du Nord-Ouest a fourni des renseignements, limités. Lorsque deux informateurs clés ont été consultés, ils ont collaboré pour répondre à un seul questionnaire d'enquête, qui a été renvoyé aux chercheurs de l'étude. Aucune réponse à l'enquête n'a été reçue du Yukon ou du Nunavut, car personne n'avait l'expertise nécessaire (la connaissance des données du registre) pour répondre aux questions. Les informateurs clés étaient des employés des ministères de la Santé provinciaux et des experts associés à des dépôts de données de recherche sur la population, comme l'ICES. La Saskatchewan et l'Îledu-Prince-Édouard ont toutes deux mentionné que le personnel responsable du registre avait été consulté pour répondre à l'enquête.

\section{Couverture temporelle des données}

Les données sur la couverture temporelle des registres d'assurance-maladie sont présentées figure 1. La première année pour laquelle des données sont disponibles est 1968, en Saskatchewan. Bien que l'informateur clé du Manitoba ait indiqué que la première année de disponibilité des données était 1984, d'autres sources publiées indiquent que les données du registre d'assurance-maladie de cette province remontent à $1970^{9,15}$. Cependant, il est probable que l'information de ces premières années ne soit pas accessible à tous les utilisateurs de données et soit difficile à utiliser lors de la production de rapports sur la surveillance et la recherche. Toutes les provinces ont précisé que des « instantanés » de leurs registres (c'est-à-dire un fichier permettant de conserver les données du registre à un

FIGURE 1

Périodes pour lesquelles des données sont disponibles dans le registre d'assurance-maladie canadien de chaque administration participante (à l'exception des Territoires du Nord-Ouest)

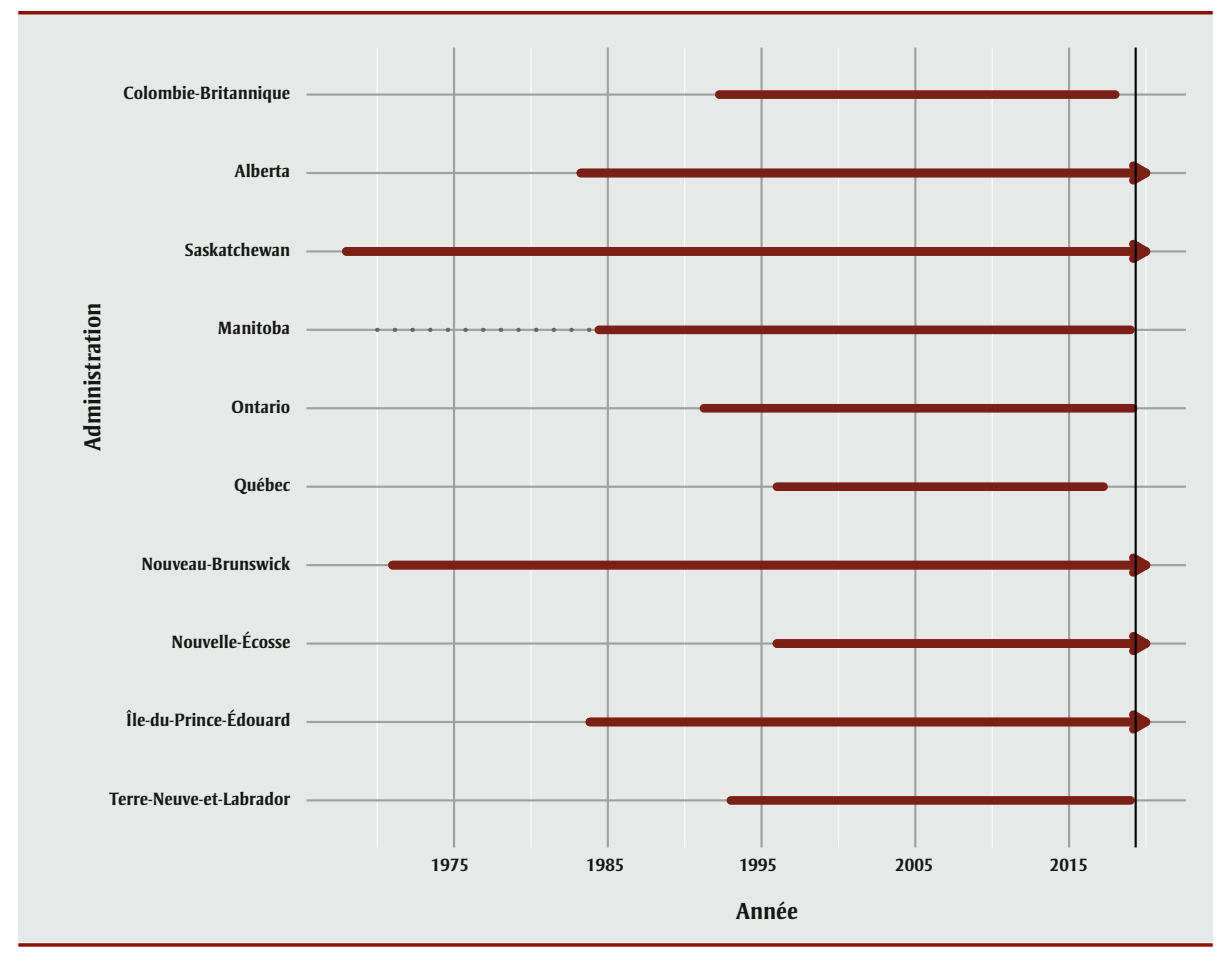

Remarques : Les flèches indiquent que les données du registre sont mises à jour quotidiennement ou hebdomadairement et que les données sont disponibles à compter de la dernière mise à jour. La ligne grise pointillée indique que les données disponibles peuvent être limitées. La ligne noire verticale indique la date de réalisation de l'enquête (c'est-à-dire avril 2019). Les périodes de disponibilité des données indiquées sont à jour en date de la réponse à l'enquête (mai 2019); des mises à jour peuvent être disponibles après cette date.

moment précis) sont régulièrement enregistrés et archivés.

\section{Début et fin de la couverture}

Le tableau 1 rassemble les réponses fournies pour les dates de début et de fin de couverture d'assurance-maladie des individus. On a observé une hétérogénéité parmi les administrations déclarantes en matière de saisie des données sur la mobilité des résidents couverts par l'assurancemaladie, c'est-à-dire l'endroit où une personne a déménagé lorsqu'elle a quitté une province ou un territoire et l'endroit d'où elle provenait lorsqu'elle est arrivée dans une province ou un territoire. Dans la plupart des provinces et territoires, les individus sont ajoutés au registre trois mois après leur arrivée et la fin de leur couverture est consignée trois mois après leur départ. Les délais d'inscription au registre et de consignation de fin de couverture varient également d'une province ou d'un territoire à l'autre dans les cas de naissance et de décès. Une grande diversité a été constatée dans les codes d'annulation de fin de couverture (personne décédée, départ du pays, dossier en double, etc.) fournis par les informateurs clés et seule la province de l'Ontario a indiqué qu'aucun code de fin de couverture n'était disponible. Bien que des contrôles de qualité des données pour le début et la fin de couverture aient été mis en œuvre dans un certain nombre de provinces (ColombieBritannique, Alberta, Saskatchewan, Ontario, Québec, Nouvelle-Écosse et Terre-Neuve-etLabrador), les informateurs clés ne disposaient pas toujours d'information sur les processus d'évaluation de la qualité des données ou n’ont pas mentionné que des évaluations en avaient été entreprises.

\section{Caractéristiques de la population et attributs saisis}

Toutes les administrations déclarantes ont mentionné que leurs registres contenaient l'adresse principale, la date de naissance et le sexe des résidents assurés (tableau 2).

L'Alberta, la Saskatchewan, le Manitoba, le Nouveau-Brunswick et les Territoires du Nord-Ouest ont fait état d'un identificateur 
TABLEAU 1

Début et fin des renseignements sur la couverture individuelle dans les registres provinciaux et territoriaux de l'assurance-maladie, Canada, 2019

\begin{tabular}{|c|c|c|c|c|c|c|c|c|c|c|c|}
\hline $\begin{array}{l}\text { Début et fin } \\
\text { de la couverture }\end{array}$ & $\begin{array}{l}\text { Colombie- } \\
\text { Britannique }\end{array}$ & Alberta & Saskatchewan & Manitoba & Ontario & Québec & $\begin{array}{l}\text { Nouveau- } \\
\text { Brunswick }\end{array}$ & Nouvelle-Écosse & $\begin{array}{l}\text { île-du-Prince- } \\
\text { Édouard }\end{array}$ & $\begin{array}{l}\text { Terre-Neuve-et- } \\
\text { Labrador }\end{array}$ & $\begin{array}{c}\text { Territoires du } \\
\text { Nord-Ouest }\end{array}$ \\
\hline \multicolumn{12}{|c|}{ Pour les résidents qui emménagent dans la province ou le territoire } \\
\hline $\begin{array}{l}\text { Lieu de départ de } \\
\text { la personne }\end{array}$ & Non & Oui & Oui & Oui & Non & Non & Oui & Oui & Oui & Oui, si au Canada & ND \\
\hline $\begin{array}{l}\text { Temps écoulé après } \\
\text { le déménagement } \\
\text { avant que les } \\
\text { nouveaux arrivants } \\
\text { (du Canada) } \\
\text { soient ajoutés au } \\
\text { registre }^{\mathrm{a}}\end{array}$ & 3 mois & $\begin{array}{l}\text { Nouveaux } \\
\text { arrivants ajoutés } \\
\text { immédiatement, } \\
\text { mais doivent } \\
\text { vivre en Alberta } \\
\text { pendant } 3 \text { mois } \\
\text { avant d'être } \\
\text { admissibles }\end{array}$ & $\begin{array}{l}3 \text { mois (c.-à-d. le } \\
\text { premier jour du } \\
\text { troisième mois } \\
\text { suivant l'arrivée) }\end{array}$ & $\begin{array}{l}\text { Nouveaux arrivants } \\
\text { ajoutés une fois la } \\
\text { demande présentée, } \\
\text { mais doivent vivre au } \\
\text { Manitoba pendant } \\
3 \text { mois (c.-à-d. le } \\
\text { premier jour du } \\
\text { troisième mois } \\
\text { complet) avant d'être }^{\text {admissibles }}{ }^{b}\end{array}$ & 3 mois & 3 mois & 90 jours & 3 mois & 3 mois & 3 mois & ND \\
\hline $\begin{array}{l}\text { Temps écoulé entre } \\
\text { le déménagement } \\
\text { et l'inscription des } \\
\text { immigrants au } \\
\text { registre }\end{array}$ & 3 mois & Variable & Variable & Incertain & 3 mois & 3 mois & 90 jours $^{c}$ & Date d'arrivée & $\begin{array}{l}\text { Premier jour } \\
\text { d'arrivée ou } \\
\text { premier jour } \\
\text { d'octroi de la } \\
\text { résidence } \\
\text { permanente, } \\
\text { selon la dernière } \\
\text { de ces deux dates }\end{array}$ & Date d'arrivée & ND \\
\hline \multicolumn{12}{|c|}{ Pour les résidents qui quittent la province ou le territoire : } \\
\hline $\begin{array}{l}\text { Endroit où la } \\
\text { personne a } \\
\text { déménagé }\end{array}$ & Non & Oui & Oui, si au Canada & Oui & Non & Non & Oui, si au Canada & Oui & $\begin{array}{l}\text { Oui, si au } \\
\text { Canada }\end{array}$ & Non & ND \\
\hline $\begin{array}{l}\text { Temps écoulé après } \\
\text { le déménagement } \\
\text { avant que la fin de } \\
\text { la couverture des } \\
\text { résidents ne soit } \\
\text { signalée }^{\mathrm{a}}\end{array}$ & $\begin{array}{l}\text { Jusqu'à ce que } \\
\text { les résidents } \\
\text { soumettent une } \\
\text { demande } \\
\text { d'annulation }\end{array}$ & $\begin{array}{l}\text { Lorsque la } \\
\text { couverture } \\
\text { commence dans } \\
\text { la nouvelle } \\
\text { province, OU le } \\
\text { jour où ils } \\
\text { quittent le pays }\end{array}$ & $\begin{array}{l}3 \text { mois (c.-à-d. } \\
\text { couvert pour le } \\
\text { reste du mois du } \\
\text { départ plus } 2 \text { mois } \\
\text { supplémentaires) }\end{array}$ & $\begin{array}{l}3 \text { mois (c.-à-d. couvert } \\
\text { pour le reste du mois } \\
\text { du départ plus } 2 \text { mois } \\
\text { supplémentaires) } \\
\text { pour les citoyens } \\
\text { canadiens et les } \\
\text { résidents permanents. } \\
\text { Jour du déménagement } \\
\text { permanent pour les } \\
\text { titulaires de permis } \\
\text { de travail et d'études }\end{array}$ & $\begin{array}{l}\text { Les données } \\
\text { ne sont pas } \\
\text { claires à ce } \\
\text { sujet }\end{array}$ & 3 mois & $\begin{array}{l}\text { Lorsque la } \\
\text { couverture } \\
\text { commence dans } \\
\text { la nouvelle province } \\
\text { OU lorsqu'on } \\
\text { avise l'assurance- } \\
\text { maladie du N.-B. } \\
\text { que la personne a } \\
\text { quitté le pays }\end{array}$ & 3 mois & $\begin{array}{l}\text { Le jour précédant } \\
\text { le début de la } \\
\text { couverture dans } \\
\text { la nouvelle } \\
\text { province }\end{array}$ & 3 mois & ND \\
\hline
\end{tabular}


TABLEAU 1 (suite)

Début et fin des renseignements sur la couverture individuelle dans les registres provinciaux et territoriaux de l'assurance-maladie, Canada, 2019

\begin{tabular}{|c|c|c|c|c|c|c|c|c|c|c|c|}
\hline $\begin{array}{l}\text { Début et fin } \\
\text { de la couverture }\end{array}$ & $\begin{array}{l}\text { Colombie- } \\
\text { Britannique }\end{array}$ & Alberta & Saskatchewan & Manitoba & Ontario & Québec & $\begin{array}{l}\text { Nouveau- } \\
\text { Brunswick }\end{array}$ & Nouvelle-Écosse & $\begin{array}{l}\text { Île-du-Prince- } \\
\text { Édouard }\end{array}$ & $\begin{array}{l}\text { Terre-Neuve-et- } \\
\text { Labrador }\end{array}$ & $\begin{array}{l}\text { Territoires du } \\
\text { Nord-Ouest }\end{array}$ \\
\hline \multicolumn{12}{|c|}{ Pour les naissances et les décès : } \\
\hline $\begin{array}{l}\text { Temps écoulé après } \\
\text { la naissance avant } \\
\text { l'ajout au registre }\end{array}$ & $\begin{array}{l}\text { Avant } 2008 \text { : } \\
\text { variable } \\
\text { Après } 2008 \text { : } \\
\text { immédiatement }\end{array}$ & $\begin{array}{l}\text { Dans un délai } \\
\text { de } 24 \text { heures }\end{array}$ & $\begin{array}{l}\text { Immédiatement } \\
\text { en date de } 1998\end{array}$ & Incertain & 3 mois & De 2 à 5 mois & $\begin{array}{l}\text { Jusqu'à ce que le } \\
\text { parent soumette } \\
\text { le formulaire } \\
\text { d'inscription } \\
\text { dûment rempli }\end{array}$ & $\begin{array}{l}\text { Généralement dans } \\
\text { les } 10 \text { jours ouvrables }\end{array}$ & $\begin{array}{l}\text { De } 24 \text { à } \\
48 \text { heures }\end{array}$ & $\begin{array}{l}\text { Jusqu'à ce que le } \\
\text { parent soumette } \\
\text { le formulaire } \\
\text { d'inscription } \\
\text { dûment rempli }\end{array}$ & ND \\
\hline $\begin{array}{l}\text { Temps écoulé après } \\
\text { le décès avant la } \\
\text { consignation du } \\
\text { décès dans le } \\
\text { registre }\end{array}$ & Incertain & Variable & Variable & Incertain & $\begin{array}{l}\text { Jusqu'à } \\
3 \text { mois }\end{array}$ & Incertain & $\begin{array}{l}\text { Les nouveaux décès } \\
\text { sont consignés } \\
\text { quotidiennement, } \\
\text { mais seront } \\
\text { confirmés par le } \\
\text { rapport bimensuel } \\
\text { des statistiques } \\
\text { de l'état civil }\end{array}$ & Variable & $\begin{array}{l}\text { De } 24 \text { à } \\
48 \text { heures }\end{array}$ & $\begin{array}{l}\text { Généralement un } \\
\text { jour après le } \\
\text { décès }\end{array}$ & ND \\
\hline $\begin{array}{l}\text { Descriptions } \\
\text { utilisées pour } \\
\text { indiquer la fin de } \\
\text { couverture dans } \\
\text { les données du } \\
\text { registre, telles que } \\
\text { fournies par } \\
\text { l'informateur }\end{array}$ & $\begin{array}{l}\text { Décédé } \\
\begin{array}{l}\text { Annulation de la } \\
\text { couverture du } \\
\text { groupe }\end{array} \\
\begin{array}{l}\text { Permis } \\
\text { temporaire } \\
\text { expiré }\end{array} \\
\text { Non participant } \\
\text { A quitté la } \\
\text { province } \\
\text { A quitté le pays }\end{array}$ & $\begin{array}{l}\text { Décédé } \\
\text { Forces armées } \\
\text { Pénitencier } \\
\text { fédéral } \\
\text { Résident illégal } \\
\text { Ajouté par erreur } \\
\text { Fraude } \\
\text { Résidence remise } \\
\text { en question - } \\
\text { politique de } \\
\text { bonne foi } \\
\text { Non participant } \\
\text { au RAMA } \\
\text { Double } \\
\text { inscription } \\
\text { A quitté l'Alb.- } \\
\text { couverture } \\
\text { normale étendue } \\
\text { A quitté l'Alb.- } \\
\text { comprend le } \\
\text { temps de } \\
\text { déplacement }\end{array}$ & $\begin{array}{l}\text { Décédé } \\
\text { Forces armées } \\
\text { canadiennes } \\
\text { Incarcéré dans un } \\
\text { établissement } \\
\text { fédéral } \\
\text { Retour du courrier } \\
\text { et adresse actuelle } \\
\text { inconnue } \\
\text { A quitté la province }\end{array}$ & $\begin{array}{l}\text { Décédé } \\
\text { Militaire/GRC } \\
\text { Détenu d'un } \\
\text { établissement fédéral } \\
\text { Inscrit par erreur } \\
\text { Impossible à } \\
\text { retrouver } \\
\text { Duplicata du NIMP } \\
\text { Couverture annulée - } \\
\text { no d'inscription }_{\text {demandé }} \\
\text { Non-résident } \\
\text { Autre (garde inconnue } \\
\text { - personne à chargé } \\
\text { mineure) } \\
\text { Résident temporaire/ } \\
\text { non Canadien } \\
\text { Adopté } \\
\text { A quitté la province - } \\
\text { lieu inconnu } \\
\text { A quitté la province - } \\
\text { T.-N.-L. }\end{array}$ & $\begin{array}{l}\text { Aucune raison } \\
\text { donnée pour } \\
\text { la fin de la } \\
\text { couverture }^{\text {d }}\end{array}$ & $\begin{array}{l}\text { Fin de l'admissibilité } \\
\text { Annulation de } \\
\text { l'assurance-maladie }\end{array}$ & $\begin{array}{l}\text { Décédé } \\
\text { Non-respect } \\
\text { Adopté } \\
\text { A quitté la province } \\
\text { A quitté le pays } \\
\text { À déterminer }\end{array}$ & $\begin{array}{l}\text { Décédé, confirmé par } \\
\text { les statistiques de } \\
\text { l'état civil } \\
\text { Décédé, famille } \\
\text { avertie } \\
\text { Fin de l'admissibilité } \\
\text { Temporairement } \\
\text { absent } \\
\text { Fin du statut d'étudiant } \\
\text { Fin de l'emploi } \\
\text { Fin de l'admissibilité } \\
\text { en raison de l'âge } \\
\text { (soins dentaire) }\end{array}$ & $\begin{array}{l}\text { Décédé } \\
\text { Gouvernement } \\
\text { fédéral } \\
\text { Pénitencier } \\
\text { fédéral } \\
\text { A quitté la } \\
\text { province }\end{array}$ & $\begin{array}{l}\text { Décédé } \\
\text { Non admissible à } \\
\text { la couverture, ou } \\
\text { Forces armées } \\
\text { Fin de la } \\
\text { couverture } \\
\text { néonatale } \\
\text { Permis de travail } \\
\text { expiré } \\
\text { Visa d'étudiant } \\
\text { expiré } \\
\text { Permis de séjour } \\
\text { expiré } \\
\text { Permis du } \\
\text { ministre expiré } \\
\text { Documents } \\
\text { d'immigration } \\
\text { expirés } \\
\text { Documents de } \\
\text { réfugié expirés } \\
\text { Enfant de } \\
\text { parents } \\
\text { immigrants }\end{array}$ & $\begin{array}{l}\text { Anscription } \\
\begin{array}{l}\text { Année } \\
\text { d'inscription } \\
\text { trop vieille }\end{array} \\
\text { Adresse inconnue } \\
\text { - déménagé } \\
\text { Inscrit par erreur } \\
\text { Immigrant } \\
\begin{array}{l}\text { Date d'expiration } \\
\text { de la carte du } \\
\text { régime de soins } \\
\text { de santé }\end{array}\end{array}$ \\
\hline
\end{tabular}

Suite à la page suivante 
TABLEAU 1 (suite)

Début et fin des renseignements sur la couverture individuelle dans les registres provinciaux et territoriaux de l'assurance-maladie, Canada, 2019

\begin{tabular}{|c|c|c|c|c|c|c|c|c|c|c|c|}
\hline $\begin{array}{l}\text { Début et fin } \\
\text { de la couverture }\end{array}$ & $\begin{array}{l}\text { Colombie- } \\
\text { Britannique }\end{array}$ & Alberta & Saskatchewan & Manitoba & Ontario & Québec & $\begin{array}{l}\text { Nouveau- } \\
\text { Brunswick }\end{array}$ & Nouvelle-Écosse & $\begin{array}{l}\text { Île-du-Prince- } \\
\text { Édouard }\end{array}$ & $\begin{array}{l}\text { Terre-Neuve-et- } \\
\text { Labrador }\end{array}$ & $\begin{array}{l}\text { Territoires du } \\
\text { Nord-Ouest }\end{array}$ \\
\hline & & $\begin{array}{l}\text { A quitté l'Alb. - } \\
\text { circonstances }\end{array}$ & & $\begin{array}{l}\text { A quitté la province - } \\
\text { Î.-P.É.E. }\end{array}$ & & & & & & $\begin{array}{l}\text { Couverture à } \\
\text { l'extérieur de la }\end{array}$ & $\begin{array}{l}\text { A omis de } \\
\text { renouveler }\end{array}$ \\
\hline & & inconnues & & A quitté la province - & & & & & & province & Carte du régime \\
\hline & & A quitté l'Alb. - & & N.-B. & & & & & & Inactif & de soins de santé \\
\hline & & $\begin{array}{l}\text { notifié par un } \\
\text { autre bureau de } \\
\text { santé provincial }\end{array}$ & & $\begin{array}{l}\text { A quitté la province - } \\
\text { N.-É. }\end{array}$ & & & & & & $\begin{array}{l}\text { A quitté la } \\
\text { province }\end{array}$ & $\begin{array}{l}\text { non réclamée/ } \\
\text { courrier non } \\
\text { livrable }\end{array}$ \\
\hline & & A quitté le & & $\begin{array}{l}\text { A quitté la province - } \\
\text { Qc. }\end{array}$ & & & & & & $\begin{array}{l}\text { A quitté la } \\
\text { province et relève }\end{array}$ & Libération d'un \\
\hline & & A quitté le & & $\begin{array}{l}\text { A quitté la province - } \\
\text { Ont. }\end{array}$ & & & & & & $\begin{array}{l}\text { des services } \\
\text { sociaux }\end{array}$ & T.N.-O. \\
\hline & & $\begin{array}{l}\text { Canada - trois } \\
\text { mois maximum }\end{array}$ & & $\begin{array}{l}\text { A quitté la province - } \\
\text { Sask. }\end{array}$ & & & & & & & $\begin{array}{l}\text { Couverture } \\
\text { suspendue }\end{array}$ \\
\hline & & A quitté le & & $\begin{array}{l}\text { A quitté la province - } \\
\text { Alb. }\end{array}$ & & & & & & & $\begin{array}{l}\text { Boîte postale } \\
\text { fermée }\end{array}$ \\
\hline & & $\begin{array}{l}\text { prolongée d'un } \\
\text { mois }\end{array}$ & & $\begin{array}{l}\text { A quitté la province - } \\
\text { C.-B. }\end{array}$ & & & & & & & $\begin{array}{l}\text { Adresse } \\
\text { incomplète }\end{array}$ \\
\hline & & $\begin{array}{l}\text { A quitté le } \\
\text { Canada - }\end{array}$ & & $\begin{array}{l}\text { A quitté la province - } \\
\text { Yn }\end{array}$ & & & & & & & $\begin{array}{l}\text { Adresse } \\
\text { inexistante }\end{array}$ \\
\hline & & $\begin{array}{l}\text { couverture } \\
\text { prolongée de }\end{array}$ & & $\begin{array}{l}\text { A quitté la province - } \\
\text { T.N.-O. }\end{array}$ & & & & & & & \\
\hline & & 3 mois & & A quitté la province - & & & & & & & \\
\hline & & Autre & & Nt & & & & & & & \\
\hline & & & & $\begin{array}{l}\text { A quitté la province - } \\
\text { États-Unis }\end{array}$ & & & & & & & \\
\hline & & & & $\begin{array}{l}\text { A quitté la province - } \\
\text { autre pays }\end{array}$ & & & & & & & \\
\hline
\end{tabular}

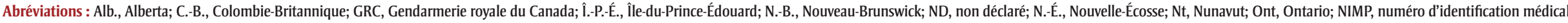
personnel; Qc, Québec; RAMA, Régime d'assurance-maladie de l'Alberta; Sask., Saskatchewan; T.-N.-L., Terre-Neuve-et-Labrador; T.N.-O., Territoires du Nord-Ouest; Yn, Territoire du Yukon.

a Les critères concernant le délai de « 3 mois » varient selon les provinces et les territoires. Des précisions ont été ajoutées lorsque les provinces et les territoires ont fourni des renseignements.

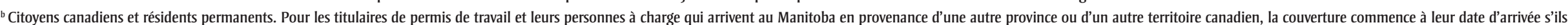
fournissent une preuve de la date d'arrivée.

' Date d'arrivée des réfugiés.

¿ Les codes de fin de couverture sont saisis par le ministère de la Santé de l'Ontario, mais ne sont pas à la disposition des détenteurs de données de recherche à l'ICES.

'Aucune activité dans le cadre du régime d'assurance-maladie.

'Lannée d'inscription indique que la couverture des soins de santé a expiré. 
TABLEAU 2

Caractéristiques de la population et attributs saisis dans les registres provinciaux et territoriaux de l'assurance-maladie, Canada, 2019

\begin{tabular}{|c|c|c|c|c|c|c|c|c|c|c|c|}
\hline $\begin{array}{c}\text { Caractéristiques de la } \\
\text { population }\end{array}$ & $\begin{array}{l}\text { Colombie- } \\
\text { Britannique }^{\mathrm{a}}\end{array}$ & Alberta & Saskatchewan & Manitoba & Ontario $^{b}$ & Québec & $\begin{array}{l}\text { Nouveau- } \\
\text { Brunswick }^{c}\end{array}$ & $\begin{array}{l}\text { Nouvelle- } \\
\text { Écosse }\end{array}$ & $\begin{array}{l}\text { Île-du-Prince- } \\
\text { Édouard }\end{array}$ & $\begin{array}{l}\text { Terre- } \\
\text { Neuve-et- } \\
\text { Labrador }\end{array}$ & $\begin{array}{c}\text { Territoires du } \\
\text { Nord-Ouest }\end{array}$ \\
\hline \multicolumn{12}{|l|}{ Résidence } \\
\hline Adresse principale & Oui & Oui & Oui & Oui & Oui & Oui & Oui & Oui & Oui & Oui & Oui \\
\hline $\begin{array}{l}\text { Adresse précédente } \\
\text { disponible? }\end{array}$ & Oui & Oui & Oui & Non & Oui & Oui & Oui & Non & Oui & Oui & Oui \\
\hline \multicolumn{12}{|l|}{ Date de naissance } \\
\hline Date de naissance & Oui & Oui & Oui & Oui & Oui & Oui & Oui & Oui & Oui & Oui & Oui \\
\hline \multicolumn{12}{|l|}{ Sexe } \\
\hline Sexe & Oui & Oui & Oui & Oui & Oui & Oui & Oui & Oui & Oui & Oui & Oui \\
\hline $\begin{array}{l}\text { Nouvelles catégorisa- } \\
\text { tions mises en œuvre }\end{array}$ & Non & Non & Non & Non & Non & Non & Oui & Oui & Non & Oui & ND \\
\hline \multicolumn{12}{|l|}{ Liens de parenté } \\
\hline $\begin{array}{l}\text { Identificateur d'unité } \\
\text { familliale }\end{array}$ & $\begin{array}{l}\text { Non, mais peut être } \\
\text { jumelé à d'autres } \\
\text { bases de données } \\
\text { pour le déterminer }\end{array}$ & Oui & Oui & Oui & Non & Non & Oui & Non & Ouie & Non & Oui \\
\hline $\begin{array}{l}\text { Liens de parenté } \\
\text { disponibles }\end{array}$ & S.o. & Unité familliale & $\begin{array}{l}\text { Chef de famille } \\
\text { (max. } 2 \text { par famille) } \\
\text { et personnes à } \\
\text { charge }\end{array}$ & $\begin{array}{l}\text { Unité familiale } \\
\text { comprenant la } \\
\text { personne inscrite } \\
\text { et, le cas échéant, } \\
\text { son conjoint et ses } \\
\text { personnes à charge }\end{array}$ & S.O. & S.O. & $\begin{array}{l}\text { Identificateur } \\
\text { "chef de } \\
\text { famille » }\end{array}$ & S.O. & ND & S.O. & ND \\
\hline
\end{tabular}


TABLEAU 2 (suite)

Caractéristiques de la population et attributs saisis dans les registres provinciaux et territoriaux de l'assurance-maladie, Canada, 2019

\begin{tabular}{|c|c|c|c|c|c|c|c|c|c|c|c|}
\hline $\begin{array}{c}\text { Caractéristiques de la } \\
\text { population }\end{array}$ & $\begin{array}{l}\text { Colombie- } \\
\text { Britannique }^{\mathrm{a}}\end{array}$ & Alberta & Saskatchewan & Manitoba & Ontariob $^{b}$ & Québec & $\begin{array}{l}\text { Nouveau- } \\
\text { Brunswick }\end{array}$ & $\begin{array}{l}\text { Nouvelle- } \\
\text { Écosse }\end{array}$ & $\begin{array}{l}\text { Île-du-Prince- } \\
\text { Édouard }\end{array}$ & $\begin{array}{c}\text { Terre- } \\
\text { Neuve-et- } \\
\text { Labrador }\end{array}$ & $\begin{array}{c}\text { Territoires du } \\
\text { Nord-Ouest }\end{array}$ \\
\hline $\begin{array}{l}\text { Codage des liens de } \\
\text { parenté }\end{array}$ & S.O. & $\begin{array}{l}\text { Les unités familiales } \\
\text { sont regroupées sous } \\
\text { un seul numéro de } \\
\text { compte } \\
\text { Codes de liens de } \\
\text { parenté particuliers : } \\
\text { Inconnu; } \\
\text { Abonné; } \\
\text { Conjoint; } \\
\text { Personne à charge } \\
\text { âgée de moins de } \\
21 \text { ans; } \\
\text { Étudiant âgé de } \\
20 \text { ans, } 8 \text { mois à } \\
25 \text { ans; } \\
\text { Personne à charge } \\
\text { permanente en } \\
\text { raison d'une } \\
\text { infirmité mentale/ } \\
\text { physique; } \\
\text { Statut d'étudiant en } \\
\text { cours d'examen }\end{array}$ & $\begin{array}{l}\text { Tous les membres } \\
\text { ont le même } \\
\text { numéro de famille } \\
\text { et les personnes } \\
\text { sont répertoriées } \\
\text { comme chef de } \\
\text { famille ou personne } \\
\text { à charge }\end{array}$ & $\begin{array}{l}\text { Tous les membres } \\
\text { de la famille } \\
\text { résidant au } \\
\text { Manitoba se voient } \\
\text { attribuer le même } \\
\text { numéro } \\
\text { d'inscription à } \\
\text { Santé Manitoba. } \\
\text { Les liens de parenté } \\
\text { particuliers sont } \\
\text { indiqués par un } \\
\text { numéro (de } 0 \text { à 8) } \\
\text { pour les personnes } \\
\text { suivantes : } \\
\text { libéré ou non } \\
\text { résident; } \\
\text { chef de famille; } \\
\text { conjoint légal; } \\
\text { conjoint de fait; } \\
\text { enfant; } \\
\text { beau-fils ou } \\
\text { belle-fille; } \\
\text { enfant invalide; } \\
\text { petit-enfant. }\end{array}$ & S.O. & S.O. & $\begin{array}{l}\text { L'identificateur } \\
\text { «chef de } \\
\text { famille » peut } \\
\text { être lié à tous } \\
\text { les membres } \\
\text { de la famille }\end{array}$ & S.O. & S.O. & S.O. & ND \\
\hline \multicolumn{12}{|l|}{ Origine ethnique } \\
\hline Origine ethnique & Non & Non & Non & Non & Oui & Non & Non & Non & Non & Non & Non \\
\hline $\begin{array}{l}\text { Origines ethniques } \\
\text { identifiables }\end{array}$ & S.O. & S.O. & S.O. & S.O. & $\begin{array}{l}\text { Généralef, } \\
\text { chinoise, } \\
\text { sud-asiatique }\end{array}$ & S.O. & S.O. & S.O. & S.O. & S.O. & S.O. \\
\hline
\end{tabular}


TABLEAU 2 (suite)

Caractéristiques de la population et attributs saisis dans les registres provinciaux et territoriaux de l'assurance-maladie, Canada, 2019

\begin{tabular}{|c|c|c|c|c|c|c|c|c|c|c|c|}
\hline $\begin{array}{c}\text { Caractéristiques de la } \\
\text { population }\end{array}$ & $\begin{array}{l}\text { Colombie- } \\
\text { Britannique }^{\mathrm{a}}\end{array}$ & Alberta & Saskatchewan & Manitoba & Ontario $^{b}$ & Québec & $\begin{array}{l}\text { Nouveau- } \\
\text { Brunswick }\end{array}$ & $\begin{array}{l}\text { Nouvelle- } \\
\text { Écosse }\end{array}$ & $\begin{array}{l}\text { Île-du-Prince- } \\
\text { Édouard }\end{array}$ & $\begin{array}{c}\text { Terre- } \\
\text { Neuve-et- } \\
\text { Labrador }\end{array}$ & $\begin{array}{c}\text { Territoires du } \\
\text { Nord-Ouest }\end{array}$ \\
\hline \multicolumn{12}{|l|}{ Statut socioéconomique } \\
\hline SSE disponible & $\begin{array}{l}\text { Non, mais peut être } \\
\text { relié à un } \\
\text { sous-ensemble de } \\
\text { données permettant } \\
\text { de le déterminer }\end{array}$ & Non & Non & Non & Oui & Oui & Non & Non & Non & Non & Non \\
\hline $\begin{array}{l}\text { Renseignements utilisés } \\
\text { pour définir le SSE }\end{array}$ & S.O. & S.O. & S.O. & S.O. & Revenus & Autre $^{8}$ & S.O. & S.O. & S.O. & S.O. & S.O. \\
\hline Codage du SSE & S.O. & S.O. & S.O. & S.O. & $\begin{array}{l}1,2,3,4,5 \\
\text { (quintiles) }\end{array}$ & $\begin{array}{l}\text { Indice de } \\
\text { défavorisa- } \\
\text { tion }\end{array}$ & S.O. & S.O. & S.O. & S.O. & S.O. \\
\hline \multicolumn{12}{|l|}{ Lieu de naissance } \\
\hline Lieu de naissance & Non & Oui & Non & Non & Non & Non & Non & $\begin{array}{l}\text { Non; peut être } \\
\text { relié à d'autres } \\
\text { bases de } \\
\text { données/ } \\
\text { sources pour } \\
\text { déterminer si } \\
\text { le lieu de } \\
\text { naissance est } \\
\text { la N.-É }\end{array}$ & Oui & $\begin{array}{l}\text { Non; peut } \\
\text { être relié à } \\
\text { d'autres } \\
\text { bases de } \\
\text { données/ } \\
\text { sources pour } \\
\text { déterminer } \\
\text { si le lieu de } \\
\text { naissance est } \\
\text { T.-N.-L. }\end{array}$ & ND \\
\hline Codage & S.O. & $\begin{array}{l}\text { Champ de texte } \\
\text { structuré }\end{array}$ & S.O. & S.O. & S.O. & S.O. & S.O. & S.O. & Pays/province & S.O. & ND \\
\hline
\end{tabular}

Abréviations : GRC, Gendarmerie royale du Canada; Man., Manitoba; ND, non déclaré; N.É., Nouvelle-Écosse; SSE, statut socioéconomique; S.O., sans objet; T.-N.L, Terre-Neuve-et-Labrador.

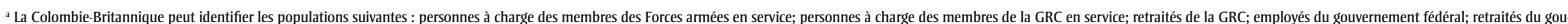
vernement fédéral; rentiers à service complet de la Colombie-Britannique; unité d'enquête des établissements correctionnels de la Colombie-Britannique; membres des Premières Nations.

${ }^{\mathrm{b}}$ Dans le cas de toutes les réponses affirmatives, les données sont obtenues par jumelagé avec d'autres bases de données - les données ne sont pas directement accessibles dans le registre.

' Le Nouveau-Brunswick est officiellement bilingue (anglais et français). Par conséquent, les données linguistiques sont également saisies dans le registre.

d « Nouvelles catégorisations mises en œuvre » désigne l'ajout d’une option « Non-binaire » en plus des catégories « Homme » et « Femme ».

e Identificateur du ménage.

${ }^{\mathrm{f}}$ Les personnes qui n’appartiennent pas à la catégorie d'origine ethnique chinoise ou sud-asiatique sont incluses dans la catégorie générale.

${ }^{8}$ Le SSE est déterminé à l'aide d'un indice de défavorisation matérielle et sociale, qui est attribué en fonction de l'emplacement géographique. 
d'unité familiale, c'est-à-dire d'un code portant sur les liens de parenté. L'Île-duPrince-Édouard a déclaré disposer d'un identificateur de ménage, mais a noté que son utilisation était limitée (par exemple les différents membres d'un foyer collectif ont le même identificateur de ménage). La Colombie-Britannique a mentionné qu'il était possible de vérifier les liens de parenté entre les résidents à partir d'autres sources de données administratives. Des codes de liens de parenté (c'est-à-dire le type de relation entre résidents, comme parent, enfant, conjoint, frère ou sœur) étaient disponibles en Alberta, en Saskatchewan, au Manitoba et au Nouveau-Brunswick.

Seule la province de l'Ontario a déclaré disposer de données sur l'origine ethnique dans son registre d'assurance-maladie, les catégories recensées en étant les suivantes : générale, chinoise et sud-asiatique. Ces catégories proviennent d'un algorithme appliqué aux données et ne sont pas directement recueillies.

Des mesures du statut socioéconomique (comme le quintile de revenu) étaient disponibles dans les registres d'assurancemaladie de l'Ontario et du Québec, celles de l'Ontario reposant sur un algorithme. La Colombie-Britannique a précisé que le statut socioéconomique pouvait être défini pour un sous-ensemble d'individus grâce au jumelage avec une autre base de données.

Enfin, le lieu de naissance était disponible dans les registres de l'Alberta et de l'îledu-Prince-Édouard, les autres provinces et territoires ayant indiqué que le jumelage des données du registre avec d'autres sources permettrait d'obtenir cette information.

\section{Information sur la population}

Le tableau 3 présente l'information disponible sur les populations à partir des registres d'assurance-maladie. Toutes les administrations déclarantes, sauf le Québec, ont dit disposer d'information sur au moins certaines populations. La qualité des variables de population était hétérogène. Par exemple, l'appartenance aux Premières Nations consignée dans le registre de l'assurance-maladie du Manitoba est susceptible de provoquer des erreurs de classification car l'information repose sur la déclaration volontaire : une personne des Premières Nations n'est identifiée comme telle que si elle divulgue cette information au personnel du registre de l'assurance-maladie. En Alberta, le gouvernement fédéral avait l'habitude de vérifier l'appartenance à une Première Nation, mais il a cessé de le faire lorsque l'Alberta a aboli les cotisations au régime d'assurancemaladie en 2009. Pour continuer à préserver la qualité des données, l’Alberta a maintenu cet indicateur pour les individus inscrits avant le changement et a attribué à leurs enfants un statut non officiel pour ses rapports. Cependant, des erreurs de classification sont susceptibles de se produire.

\section{Analyse}

Les résultats de l'enquête fournissent une information importante sur les données des registres d'assurance-maladie au Canada, pour 10 provinces et pour les Territoires du Nord-Ouest. Les données des registres de l'assurance-maladie remontent à 1968 , dans le cas de la Saskatchewan, toutes les autres administrations déclarantes disposant de données remontant au moins à 1996. L'ajout au registre et la consignation de la fin de couverture des individus qui arrivent dans la province ou le territoire et en repartent sont faits trois mois après le déménagement, les délais étant davantage variables dans le cas du début ou de la fin de la couverture découlant d'une naissance ou d'un décès. L'information enregistrée pour la fin de couverture diffère d'une province ou territoire à l'autre. Toutes les administrations déclarantes enregistrent l'adresse principale, la date de naissance et le sexe. Les identifiants familiaux, l'origine ethnique, le statut socioéconomique et le lieu de naissance sont quant à eux enregistrés dans certaines administrations déclarantes seulement. La capacité à identifier les attributs de certaines populations, comme l'appartenance aux Premières Nations et aux Forces armées canadiennes ou le fait d'être détenu dans un pénitencier fédéral varie selon les provinces et territoires.

Des recherches antérieures ont permis de comparer l'information obtenue à partir des bases de données sur les services médicaux au Canada ${ }^{16}$. En revanche, peu de recherches ont été menées sur les données des registres d'assurance-maladie provinciaux et territoriaux et sur leur qualité. Des publications sur les données des registres ont mentionné les registres d'assurancemaladie du Manitoba et de la ColombieBritannique ${ }^{9,11,17}$, mais la publication manitobaine la plus récente remonte à $1999^{\circ}$ et l'article sur la Colombie-Britannique portait sur l'élaboration d'un registre de recherche auquel le registre d'assurance-maladie provincial avait contribué ${ }^{17}$. Tang et ses collaborateurs $^{18}$ ont fait état de catégorisations sur l'origine ethnique disponibles dans les registres d'assurance-maladie canadiens et ont indiqué que les registres mentionnaient uniquement l'appartenance aux Premières Nations. Cependant, l'Ontario dispose d'indicateurs issus d'un algorithme pour les sous-populations d'origine sud-asiatique et chinoise. Un examen systématique mené par Hinds et ses collaborateurs $^{13}$ n'a pas relevé d'études ayant porté sur la qualité des données des registres d'assurance-maladie.

La saisie des caractéristiques et des attributs de la population permet de stratifier les mesures de surveillance en fonction des facteurs de risque (que sont par exemple les déterminants sociaux de la santé comme le statut socioéconomique, le statut vis-à-vis de l'immigration et l'origine ethnique). Les résultats présentés ici permettent de savoir dans quelles provinces et quels territoires ces analyses sont possibles. L'hétérogénéité au sein des provinces et territoires dans la caractérisation de la population a également des répercussions sur le plan de l'inclusion des individus dans les estimations du SCSMC, les inclusions et exclusions n'étant pas forcément uniformes.

L'un des principaux atouts du registre de l'assurance-maladie est la conservation d'instantanés, ou d'enregistrements horodatés, de ses données ${ }^{9}$. Les résultats de notre étude indiquent que plus de 20 ans de données de registre sont disponibles dans toutes les administrations déclarantes et que des instantanés sont disponibles pour saisir les changements apportés au registre. Toutefois, l'utilisation des registres d'assurance-maladie pour les études longitudinales présente des difficultés : ils ne sont pas statiques, les données et leur qualité changeant au fil du temps, et il n'est pas toujours possible de jumeler les données anciennes. Néanmoins, les avantages de pouvoir utiliser ces données pour mener des études longitudinales de cohorte et intergénérationnelles à faible coût et avec une collecte de données minimale l'emportent largement sur les inconvénients.

\section{Forces et limites}

Cette étude est exceptionnelle dans la mesure où elle dresse un portrait de l'information disponible dans les registres 
TABLEAU 3

Information sur la population disponible dans les registres provinciaux et territoriaux de l'assurance-maladie, Canada, 2019

\begin{tabular}{|c|c|c|c|c|c|c|c|c|c|c|c|}
\hline $\begin{array}{l}\text { Identification de la } \\
\text { population }\end{array}$ & $\begin{array}{l}\text { Colombie- } \\
\text { Britannique }^{\mathrm{a}}\end{array}$ & Alberta & Saskatchewan & Manitoba & Ontario $^{\mathrm{j}}$ & Québec & $\begin{array}{l}\text { Nouveau- } \\
\text { Brunswick }^{k}\end{array}$ & Nouvelle-Écosse & $\begin{array}{l}\text { île-du- } \\
\text { Prince- } \\
\text { Édouard }\end{array}$ & $\begin{array}{l}\text { Terre-Neuve-et- } \\
\text { Labrador }\end{array}$ & $\begin{array}{c}\text { Territoires du } \\
\text { Nord-Ouest }\end{array}$ \\
\hline \multicolumn{12}{|l|}{ Population } \\
\hline $\begin{array}{l}\text { Membres à temps } \\
\text { plein des Forces } \\
\text { armées canadiennes }\end{array}$ & ND & Oui & Ouid $^{d}$ & Ouis & Non & Non & Ouil & Non $^{0}$ & Non & Non & ND \\
\hline $\begin{array}{l}\text { Membres à temps } \\
\text { partiel des Forces } \\
\text { armées canadiennes }\end{array}$ & ND & Non & Ouid $^{\text {d }}$ & Oui ${ }^{8}$ & Non & Non & Non & Non & Non & Non & ND \\
\hline Anciens combattants & Non & Oui & Non & ND & Non & Non & Non & Non $^{\circ}$ & Non & Non & ND \\
\hline $\begin{array}{l}\text { Gendarmerie royale } \\
\text { du Canada }\end{array}$ & Non & Oui & Non & Oui & Non & Non & Oui & Non $^{0}$ & Non & Non & ND \\
\hline $\begin{array}{l}\text { Détenus d'un } \\
\text { pénitencier fédéral }\end{array}$ & ND & Oui & Ouie & Oui $^{\text {h }}$ & Non & Non & Non & Non & Oui & Non & ND \\
\hline $\begin{array}{l}\text { Détenus d'un } \\
\text { pénitencier provincial }\end{array}$ & Oui & Oui $^{b}$ & Oui & ND & Non & Non & Oui & Non & Non & Non & ND \\
\hline $\begin{array}{l}\text { Premières Nations } \\
\text { dans les réserves }\end{array}$ & ND & Oui $^{b}$ & Ouif $^{\mathrm{f}}$ & Ouii & Oui & Non & Non $^{m}$ & Non & Non & Non & Oui \\
\hline $\begin{array}{l}\text { Premières Nations } \\
\text { hors réserve }\end{array}$ & ND & Oui $^{b}$ & Ouif & Ouii & Oui & Non & Non $^{m}$ & Non & Non & Non & Oui \\
\hline Inuit & Non & Ouib $^{b}$ & Non & ND & Oui & Non & Non & Non & Non & Non & Oui \\
\hline Métis & Non & Non $^{c}$ & Non & ND & Oui & Non & Non & Non & Non & Non & Oui \\
\hline \multicolumn{12}{|c|}{ Résidents non permanents } \\
\hline Demandeurs d'asile & Oui & Oui & Oui & ND & Oui & Non & Non & Non & Oui & $\begin{array}{l}\text { Oui, à la fin de } \\
\text { la couverture }\end{array}$ & ND \\
\hline $\begin{array}{l}\text { Personnes ayant un } \\
\text { permis d'études }\end{array}$ & Non & Oui & Oui & ND & Non & Non & Oui & Oui & Oui & $\begin{array}{l}\text { Oui, à la fin de } \\
\text { la couverture }\end{array}$ & ND \\
\hline $\begin{array}{l}\text { Personnes ayant un } \\
\text { permis de travail }\end{array}$ & Non & Oui & Oui & ND & Non & Non & Non & Oui & Oui & $\begin{array}{l}\text { Oui, à la fin de } \\
\text { la couverture }\end{array}$ & ND \\
\hline
\end{tabular}

Suite à la page suivante 
TABLEAU 3 (suite)

Information sur la population disponible dans les registres provinciaux et territoriaux de l'assurance-maladie, Canada, 2019

\begin{tabular}{|c|c|c|c|c|c|c|c|c|c|c|c|}
\hline $\begin{array}{l}\text { Identification de la } \\
\text { population }\end{array}$ & $\begin{array}{l}\text { Colombie- } \\
\text { Britannique }^{\mathrm{a}}\end{array}$ & Alberta & Saskatchewan & Manitoba & Ontario & Québec & $\begin{array}{c}\text { Nouveau- } \\
\text { Brunswick }^{k}\end{array}$ & Nouvelle-Écosse & $\begin{array}{l}\text { île-du- } \\
\text { Prince- } \\
\text { Édouard }\end{array}$ & $\begin{array}{l}\text { Terre-Neuve-et- } \\
\text { Labrador }\end{array}$ & $\begin{array}{c}\text { Territoires du } \\
\text { Nord-Ouest }\end{array}$ \\
\hline \multicolumn{12}{|l|}{ Immigrants } \\
\hline Immigrants reçus & ND & Oui & Oui & ND & Oui & Non & Ouin $^{n}$ & Oui & Oui & $\begin{array}{l}\text { Oui, à la fin de } \\
\text { la couverture }\end{array}$ & ND \\
\hline \multicolumn{12}{|l|}{ Résidents permanents } \\
\hline $\begin{array}{l}\text { Immigrants } \\
\text { économiques }\end{array}$ & Non & Non & Non & ND & Oui & Non & Non & Non & Oui & Non & ND \\
\hline $\begin{array}{l}\text { Membres de la } \\
\text { famille des résidents } \\
\text { permanents }\end{array}$ & ND & Oui & Non & ND & Non & Non & Non & Non & Oui & Non & ND \\
\hline
\end{tabular}

Abréviations : GRC, Gendarmerie royale du Canada; ND, non déclaré.

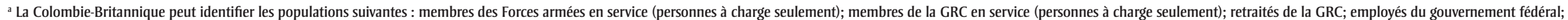
retraités du gouvernement fédéral; rentiers à service complet de la Colombie-Britannique; unité d'enquête des établissements correctionnels de la Colombie-Britannique; membres des Premières Nations.

${ }^{\mathrm{b}}$ La saisie active de cet indicateur a pris fin en janvier 2009.

' Il est possible d'établir un lien avec une base de données pour déterminer le statut à des fins d'analyse.

${ }^{\mathrm{d}}$ II n’y a pas de distinction entre les membres des Forces armées à temps partiel et à temps plein.

${ }^{e}$ Signalé seulement si une couverture était fournie par la Saskatchewan avant l'incarcération.

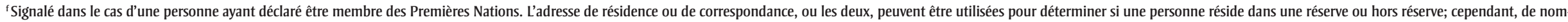
breuses adresses peuvent être ambiguës lorsqu'il s’agit de déterminer si le lieu de résidence est dans une réserve ou hors réserve.

8 II n’y a pas de distinction entre les membres des Forces armées à temps partiel et à temps plein.

h On ne peut voir que les personnes dont la couverture a été annulée pour le motif « Code 8 - Détenu d’un établissement fédéral ».

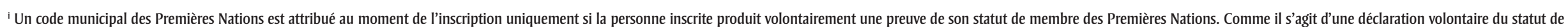
membre des Premières Nations, on estime généralement qu'environ 60 \% seulement de la population des Premières Nations du Manitoba est identifiée comme telle dans le registre d'assurance-maladie du Manitoba.

j Dans le cas de toutes les réponses affirmatives, les données sont obtenues par jumelage entre les données du registre et celles d’autres bases de données; les données ne sont pas directement accessibles dans le registre.

${ }^{k}$ Le Nouveau-Brunswick est officiellement bilingue (anglais et français). Par conséquent, les données linguistiques sont saisies dans le registre.

' Certains membres à temps plein sont identifiés, mais pas tous.

m Depuis février 2020, le ministère de la Santé du Nouveau-Brunswick collabore avec les Premières Nations en vue de créer un identificateur des Premières Nations.

"II n'y a pas de distinction entre les immigrants reçus et les résidents permanents dans le système d'assurance-maladie du Nouveau-Brunswick.

• Indiqué seulement si la personne l'a déclaré. Ne représente pas une source fiable d'information sur la population, car ces renseignements ne sont pas complets. 
d'assurance-maladie à l'échelle du Canada, au moyen d'une enquête normalisée couvrant la presque totalité des provinces et des territoires. Ce sont des personnes ayant une connaissance approfondie des données du registre et ayant accès à d'autres informateurs pour fournir des renseignements supplémentaires, au besoin, qui ont répondu à l'enquête.

Cependant, l'étude comporte certaines limites. Nous n'avons pas eu recours à une enquête validée pour recueillir l'information, car ce type enquête n'existe pas, de sorte qu'il est impossible d'établir des comparaisons avec d'autres pays. De plus, les registres d'assurance-maladie sont des bases de données complexes, et il n'est pas possible de saisir toutes les nuances de leurs caractéristiques dans une enquête menée à un moment précis dans le temps. Les éléments recueillis au cours du processus d'inscription ne seront pas tous à la disposition des utilisateurs potentiels de données. Bon nombre de ces éléments sont de nature administrative. De nombreux systèmes sont dynamiques et font l'objet d'une mise à jour permanente. C'est pourquoi des extraits ou des instantanés sont créés, à l'aide d'une méthodologie visant à offrir la vue la plus précise et la plus cohérente possible de la population.

\section{Recherches à venir}

D'autres études pourraient être entreprises pour valider les principales caractéristiques de la population incluses dans les données des registres d'assurance-maladie, notamment les dates de naissance et de décès, les codes d'annulation de couverture, le lieu de résidence et l'information sur les populations particulières. Une autre possibilité de recherche réside dans l'évaluation de la rapidité des mises à jour des caractéristiques. Cependant, l'un des principaux défis de ces études est de trouver des sources de données de validation appropriées et accessibles, disponibles à l'échelle de la population et qu'on puisse jumeler aux données d'inscription à l'assurance-maladie. Alors que les données de l'état civil sont utilisables pour évaluer l'exactitude des dates de naissance et de décès, les sources de données de validation liées aux caractéristiques clés de la population ne sont pas toujours facilement disponibles dans toutes les provinces et tous les territoires.
Des études antérieures ont comparé les données de population agrégées obtenues à partir des registres d'assurance-maladie aux données provenant des recensements de Statistique Canada ${ }^{19,20}$. Cependant, comme les sources potentielles d'inexactitudes n’ont pas été déterminées, de futures recherches pourraient examiner les sources potentielles de divergence entre les données de population dans les registres et celles du recensement et estimer l'incidence de ces divergences sur les mesures de la santé.

L'étude des changements dans la couverture en ce qui concerne l'inscription à l'assurance-maladie au fil du temps est un autre domaine potentiel de recherche. Les registres ne sont pas statiques et les exclusions ne sont peut-être pas uniformes au fil du temps. Une information exhaustive sur les changements dans les données va pouvoir garantir l'exactitude des estimations des tendances en matière de santé.

Les recherches futures devraient étudier également la faisabilité d'un cadre de données commun, s'inspirant par exemple du Modèle de données généralisé proposé par Danese et ses collaborateurs ${ }^{21}$ pour faciliter la saisie systématique et normalisée de l'information dans les registres d'assurancemaladie. Cependant, l'accès à certains éléments de données du registre est assujetti à la législation sur la protection des renseignements personnels, les données étant principalement recueillies à des fins administratives et non de recherche. La mise en œuvre d'un cadre de données commun est donc susceptible d'entraîner certaines difficultés.

Enfin, certains organismes nationaux pourraient contribuer à faciliter l'harmonisation et la normalisation des données dans les registres d'assurance-maladie. Parmi ces organismes, citons l'Institut canadien d'information sur la santé, qui a récemment proposé des normes pour la collecte de données sur l'origine ethnique des patients $^{22}$, et le Réseau de recherche sur les données de santé du Canada, qui vise à soutenir les études intergouvernementales en établissant des liens au moyen d'une infrastructure de données de recherche ${ }^{23}$.

\section{Remerciements}

Ces travaux ont pu être réalisés grâce à la collaboration entre l'ASPC et les gouvernements provinciaux et territoriaux de la
Colombie-Britannique, de l'Alberta, de la Saskatchewan, du Manitoba, de l'Ontario, du Québec, du Nouveau-Brunswick, de la Nouvelle-Écosse, de l'Île-du-Prince-Édouard, de Terre-Neuve-et-Labrador et du Yukon. Il ne faut pas inférer qu'ils ont été sanctionnés par les provinces et les territoires.

\section{Conflits d'intérêts}

Les auteurs déclarent n'avoir aucun conflit d'intérêts.

\section{Contributions des auteurs et avis}

$\mathrm{NCH}$ et LML ont rédigé le manuscrit et tous les auteurs ont contribué à sa révision. LML, CR, JE, SO, LM et KH ont défini la portée de l'enquête. NCH, LML, $\mathrm{CR}$, JE, SO, LM et $\mathrm{KH}$ ont élaboré l'enquête. LML et $\mathrm{NCH}$ ont communiqué avec les informateurs clés et ont recueilli les réponses à l'enquête. LR, KAMP, MA, MS, RP, MM, AY, JS, YL, LWS, FS, AA, BZ et JA ont vérifié l'exactitude du manuscrit et se sont assurés de la bonne interprétation des données des registres d'assurancemaladie.

Le contenu de cet article et les points de vue qui y sont exprimés n'engagent que les auteurs; ils ne correspondent pas nécessairement à ceux du gouvernement du Canada.

\section{Références}

1. LeBlanc AG, Gao YJ, McRae L, Pelletier C. Aperçu - Vingt ans de surveillance du diabète grâce au Système canadien de surveillance des maladies chroniques. Promotion de la santé et prévention des maladies chroniques au Canada. 2019;39(11): 333-337.

2. Robitaille C, Dai S, Waters C, et al. Diagnosed hypertension in Canada: incidence, prevalence and associated mortality. CMAJ. 2012;184(1):E49-E56. https://doi.org/10.1503/cmaj.101863

3. Robitaille C, Bancej C, Dai S, et al. Surveillance of ischemic heart disease should include physician billing claims: population-based evidence from administrative health data across seven Canadian provinces. BMC Cardiovasc Disord. 2013;13:88. https://doi.org /10.1186/1471-2261-13-88 
4. Blais C, Dai S, Waters C, et al. Assessing the burden of hospitalized and community-care heart failure in Canada. Can J Cardiol. 2014;30(3): 352-358. https://doi.org/10.1016/j.cjca .2013 .12 .013

5. Blais C, Jean S, Sirois C, et al. Le Système intégré de surveillance des maladies chroniques du Québec (SISMACQ), une approche novatrice. Maladies chroniques et blessures au Canada. 2014;34(4):247-256.

6. Gershon AS, Guan J, Wang C, To T. Trends in asthma prevalence and incidence in Ontario, Canada, 1996-2005: a population study. Am J Epidemiol. 2010;172(6):728-736. https://doi.org /10.1093/aje/kwq189

7. Hux JE, Ivis F, Flintoft V, Bica A. Diabetes in Ontario: determination of prevalence and incidence using a validated administrative data algorithm. Diabetes Care. 2002;25(3):512-516. https://doi.org/10.2337/diacare.25.3 .512

8. Lix L, Ayles J, Bartholomew S, et al. The Canadian Chronic Disease Surveillance System: a model for collaborative surveillance. Int $\mathrm{J}$ Popul Data Sci. 2018;3(3):433. https://doi.org/10 .23889/ijpds.v3i3.433

9. Roos LL, Nicol JP. A research registry: uses, development, and accuracy. J Clin Epidemiol. 1999;52(1):39-47. https:// doi.org/10.1016/s0895-4356(98)00126-7

10. Santé Canada. Le système des soins de santé du Canada [Internet]. Ottawa (Ont.) : Gouvernement du Canada; 2011 [consultation le 23 juillet 2020]. En ligne à : https://www.canada.ca/fr /sante-canada/services/systeme-soins -sante/rapports-publications/regime -soins-sante/canada.html

11. Roos LL, Mustard CA, Nicol JP, et al. Registries and administrative data: organization and accuracy. Med Care. 1993;31(3):201-212. https://doi.org/10 $.1097 / 00005650-199303000-00002$

12. Roos LL, Gupta S, Soodeen R-A, Jebamani L. Data quality in an information-rich environment: Canada as an example. Can J Aging. 2005;24 Suppl 1:153-170. https://doi.org/10 .1353/cja.2005.0055
13. Hinds A, Lix LM, Smith M, Quan H, Sanmartin C. Quality of administrative health databases in Canada: a scoping review. Can J Public Health. 2016;107(1):e56-e61. https://doi.org /10.17269/cjph.107.5244

14. University of Manitoba Office of Research Ethics \& Compliance. Research requiring ethics review [Internet]. Winnipeg (MB) : University of Manitoba; 2019 [consultation le 23 juillet 2020]. En ligne à : https://www.umanitoba .ca/research/orec/ethics_medicine /requiring_review.html

15. Manitoba Centre for Health Policy. Manitoba population research data repository data descriptions [Internet]. Winnipeg (MB) : University of Manitoba; 2020 [consultation le 6 mai 2021]. En ligne à : https://umanitoba.ca/faculties /health_sciences/medicine/units /chs/departmental_units/mchp /resources/repository/descriptions .html?ds = Insurance

16. Lix LM, Walker R, Quan H, Nesdole $\mathrm{R}$, Yang J, Chen G. Caractéristiques des bases de données sur les services médicaux au Canada. Maladies chroniques et blessures au Canada. 2012; $32(4): 207-215$.

17. Broemeling A-M, Kerluke K, Black C, Peterson S, MacDonald A, McKendry R. Developing and maintaining a population research registry to support primary healthcare research. Healthc Policy. 2009;5(Spec No):65-76.

18. Tang K, Lucyk K, Quan H. Canadian data sources on ethnic classifications [abstract]. J Gen Intern Med. 2016 May;31(Suppl 2):S144-S145.

19. Ellison J, Nagamuthu C, Vanderloo S, McRae B, Waters C. Taux de maladies chroniques au Canada : quel dénominateur utiliser pour l'estimation de l'ensemble de la population? Promotion de la santé et prévention des maladies chroniques au Canada. 2016; 36(10):250-257. https://doi.org/10.24095 /hpcdp.36.10.03f

20. Jin Y. Comparison of Alberta population counts between the AHCIP Registry and the 2006 Census. Edmonton (AB) : Alberta Health and Wellness; 2009. En ligne à : https://open.alberta.ca /publications/9780778582502\#detailed
21. Danese MD, Halperin M, Duryea J, Duryea R. The Generalized Data Model for clinical research. BMC Med Inform Decis Mak. 2019;19(1):117. https://doi.org/10.1186/s12911-019 $-0837-5$

22. Institut canadien d'information sur la santé (ICIS). Normes proposées pour la collecte de données et la production de rapports sur la santé fondées sur la race et l'identité autochtone au Canada. Ottawa (Ont.) : ICIS; 2020.

23. Réseau de recherche sur les données de santé du Canada. À propos [Internet]. Vancouver (C.-B.) : Réseau de recherche sur les données de santé du Canada; 2020 [consulté le 28 septembre 2020]. En ligne à : https:// www.hdrn.ca/fr/propos 